\title{
A Graphical Model for Human Activity Recognition
}

\author{
Rocío Díaz de León ${ }^{1}$ and Luis Enrique Sucar ${ }^{2}$ \\ 1 IPICyT, Camino a la Presa San Jose 2025 \\ San Luis Potosí, S.L.P. 78216, México \\ rdiaz@ipicyt.edu.mx \\ 2 ITESM Cuernavaca, Reforma 182-A, Colonia Palmira \\ Cuernavaca, Morelos 62589, México \\ esucar@itesm.mx
}

\begin{abstract}
We propose a general model for visual recognition of human activities, based on a probabilistic graphical framework. The motion of each limb and the coordination between them is considered in a layered network that can represent and recognize a wide range of human activities. By using this model and a sliding window, we can recognize simultaneous activities in a continuous way. We explore two inference methods for obtaining the most probable set of activities per window: probability propagation and abduction. In contrast with the standard approach that uses several models, we use a single classifier for multiple activity recognition. We evaluated the model with real image sequences of 6 different activities performed continuously by different people. The experiments show high recall and recognition rates.
\end{abstract}

\section{Introduction}

The recognition of human activities from video sequences is an important problem with many applications, such as surveillance, human-computer interaction, sports training, and rehabilitation. Recently there has been an increasing amount of work in this area, but there are still several outstanding issues to be solved: (i) continuous recognition, (ii) simultaneous activities, (iii) occlusions, (iv) scale and view variations. Existing models are targeted for certain types of activities or specific applications, so there is no general, high-level model for activity representation and recognition. The main hypothesis of this work is that most human activities can be recognized based on the motion of the limbs, and the relations between them.

We propose a general model to represent and recognize human activities, based on a layered, probabilistic graphical network, that considers the motion of the limbs' and their dependencies. The top layer represents the different activities, and the bottom layer the motion of each limb. The intermediate layer considers a classification of the limbs' motions, so these can be interpreted as different modalities for an activity. The structure of the model reflects the dependencies of each activity on the limbs' motions, and their coordination. The 
model includes several outstanding aspects: (i) representation of different activities using one model, (ii) recognition of simultaneous activities, (iii) recognition of activities performed on a continuous way. Using this model we can represent and recognize a wide range of human activities.

A Bayesian network based on the general model was implemented and tested with real image sequences of different activities: (i) activities performed with all limbs (arms and legs) at the same time like aerobics; (ii) activities performed with just one limb (right arm) like goodbye and right; and (iii) activities performed with two limbs at the same time like attracting the attention (both arms), and jump and walk (both legs). In some test sequences several activities were performed at the same time, for example, jump and attracting the attention. Recognition was performed using two alternative inference methods: likelihood weighting and total abduction. The model was tested with video sequences of different people, with a high recall-precision for both inference schemes.

\section{Related Work}

Several computational models for human activity recognition have been proposed, including neural networks [3], hidden Markov models [10], Bayesian networks [2], scale-space curve analysis [9], spatio-temporal models [8,12] and exemplars based on principal components [11].

Vogler and Metaxas [10] use parallel hidden Markov models to recognize American sign language. They consider signs that are performed with one or both hands. They assume that the motion of each limb evolves independently, while we consider activities that require coordination. [2] use DBNs for hand gesture recognition. With dynamic models, such as HMMs and DBNs, it is difficult to perform temporal segmentation for continuous recognition.

[9] present a view invariant representation using the spatio-temporal curvature of the trajectory of the hand. This model is restricted to activities performed with one limb. [8] describes a system that uses periodicity measures of the image sequences for recognizing periodic activities; it requires at least 4 repetitions for recognition. A model based on parameterized exemplars was developed by [11], and recognition involves matching the observation against the exemplars using principal component analysis. The previous models assume a previous segmentation of the activities, so they can not recognize continuous activities.

There are very few systems that can recognize activities performed in a continuous way. [1] describes a model to represent and recognize continuous activities. Their model represents 7 activities performed with the legs and the torso. They use a nearest neighbor classifier obtaining an average recognition rate of $77 \%$. They comment that tracking the trajectory of other parts of the body along with the leg components could make the system more robust, and this would help in recognizing more complex actions taken from different views. [12] constructs an event based representation of a video sequence using spatio-temporal templates at different scales, and use a sliding temporal window for continuous recognition. However, this approach recognizes activities based on the global motion, so it can not differentiate similar or simultaneous activities. 


\section{Recognition Process}

Our recognition process involves the following stages:

VIDEO $\rightarrow$ partition $\rightarrow$ feature extraction $\rightarrow$ recognition $\rightarrow$ ACTIVITIES

The video sequence is partitioned in a series of overlapping windows, with $N$ frames per window, and an overlap of $N-3$ frames. For each consecutive image pair in a window, we obtain the motion parameters of each limb (arms and legs), which are transformed into a discretized representation which we call displacement directions. The displacement directions are reduced to consider only a fixed number of different displacement directions in a window, and these constitute the feature set per limb, in each window, that is fed to the recognition network. Based on these features, the recognition process determines the most probable activities in each window via probability propagation.

\subsection{Feature Extraction}

The first step in the recognition process is to obtain the motion features of the different parts of the body. Although there could be global motion of the person or other parts of the body, we consider that the motions of the limbs are the most relevant features for human activities. The global trajectory of a limb is its position sequence $(X, Y, Z)$ when the activity is performed. Given that our current focus is on the high-level recognition model, we simplify the feature extraction process by using color landmarks on the wrists and the ankles. We apply a color detection process to get each landmark based on a Bayesian classifier [4]. We segment the landmarks using 8-connectivity and we extract their center of mass (we initially consider only $X, Y$ ) to get the wrist and the ankle positions in each frame; these are tracked in the image sequence.

Working with the absolute $X, Y$ positions of the landmarks' centroids is very susceptible to the distance between the person and the camera, and her way of performing the activity. The angular velocity of each limb is a more robust feature for recognition. Therefore, we estimate the angular velocity by obtaining the displacement direction (dd), for each limb, between two consecutive frames as: $d d(i)=\arctan ((y 2-y 1) /(x 2-x 1))$; where $x 1, y 1$ are centroid coordinates for limb(i) in frame $t$, and $x 2, y 2$ for limb(i) in frame $t+1$. The displacement directions are discretized in eight 45 degree intervals ( 8 values). When there is no movement between frames we assign a value of zero. Although we are currently using markers, the estimate of the angular motion of each limb can be obtained using low-level vision techniques, for instance motion [11] or color [9].

The problem is how to determine how many frames are required to represent an activity. For this we consider that most human activities are periodic, such as walking, running, waving the hand, etc. So an activity can be characterized by a basic pattern that is repeated. In many activities, this basic pattern has a relative short duration, usually less than a second. Thus, we use a one second window, with 15 images per window (frame rate is 15 images per second). In principle, we can have 15 displacement directions in a window. However, we notice that many times the $d d$ is the same for several consecutive frames. So instead of using 
all the $d d$ in a window, we only use the different displacement directions (ddd). Based on statistics from different activities performed by different people, we obtained that in a sequence of 15 frames, 5 different displacement directions are enough to represent the basic pattern of an activity. So we look for at most 5 different displacement directions per window. If more than 5 different directions are detected in the window, we only consider the first five; and if there are less than 5, the rest are set to zero. These features are used in the recognition network. The observation window is continuously taken from the image sequence by displacing the window 3 frames each step, until the complete sequence is scanned. Key frames from a video are shown in figure 1.

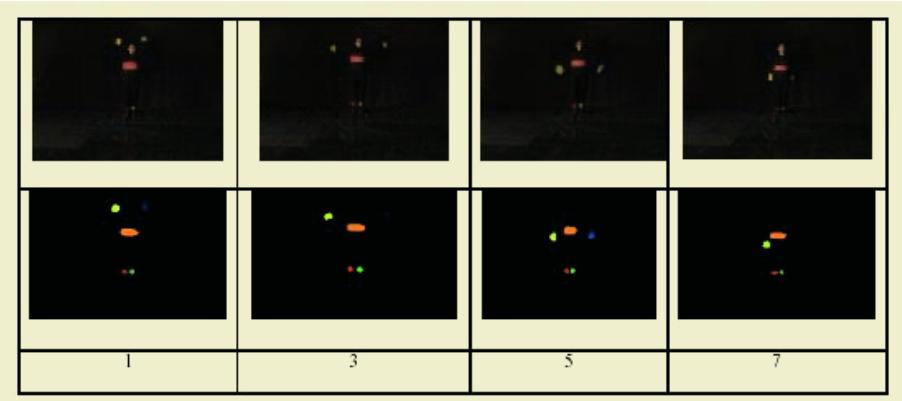

Fig. 1. Part of the image sequence for two simultaneous activities, jump and attract the attention. Top row: original images. Middle row: landmarks. Bottom row: frame number. There are 5 landmarks, we only use 4 (ankles and wrists) for the experiments.

\section{Recognition Model}

Our recognition model is based on the assumption that many human activities can be recognized by the motion of the limbs. It considers that several activities can be executed simultaneously. It also takes into account the inherent uncertainty in the model due to variations in activity execution and to the feature extraction process.

We propose a model to represent human activities based on a Bayesian network classifier. The model represents each activity and each limb as a variable. Each activity variable is related to the limbs' variables that are relevant for this activity. Each limb variable is related to the motion features (ddd) for that limb, and to other limbs, taking into account the dependencies between limbs' motions. The Bayesian network model (fig. 2) includes 3 layers:

- Top layer (root nodes): a binary node for each activity, $A_{i}$

- Middle layer: a multivalued node for each limb, $L_{i}$

- Bottom layer (leaf nodes): motion information for each limb ( $m$ node, one for each $d d d$ in a window), $D_{i}$.

The model considers that each activity is recognized by combining the motion features of the limbs. Depending on the activity, different limbs are considered. 


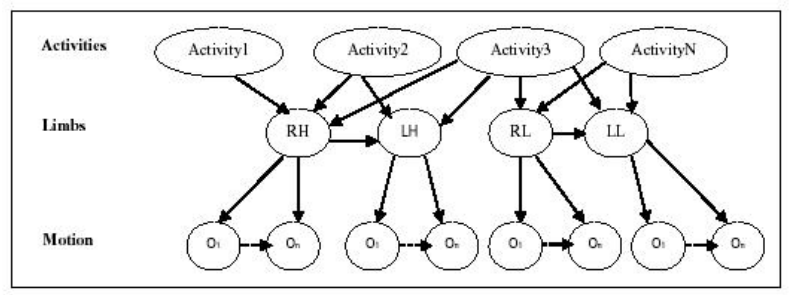

Fig. 2. General recognition model. The top layer nodes correspond to activities, $A_{i}$, the middle to limbs, $L_{i}$, and the bottom to observations. The observations, $O_{1: n}$, per limb, are the $d d d, D_{i}$, obtained in the feature extraction process.

The nodes in the middle level give information about the type of movement of each limb. The coordination between limb motions is considered in this case by the link between hand nodes (LH-RH) and the leg nodes (LL-RL). However, it is possible to consider also the dependencies between hands and legs. The leaf nodes are linked in a dynamic chain, so that each $d d d$ depends on the previous one (except the first one in the window). Thus, the network can be used to complete the trajectory in case there is missing information (occlusion), providing the most probable trajectory.

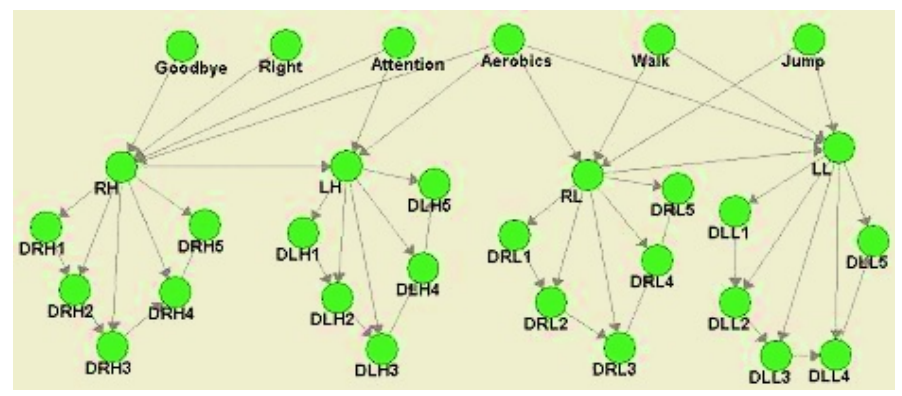

Fig. 3. Recognition network used in the experiments.

The BN in figure 3 represents a particular case of the general model which was used in the experiments. This network considers 6 activities: goodbye, move right, attracting the attention, walk, jump and aerobics; and 5 direction changes per window. The intermediate or limb nodes (RH,LH,RL,LL) are used to represent the type of motion performed by each limb. Each limb node has several possible values, associated to different activities, so we can have different descriptions for each activity. In our test network, the activity walk, has two subtypes, walk in front of, walk in profile, that are expressed by the intermediate nodes; so these can also be recognized. 


\subsection{Learning}

Learning a Bayesian network can be divided into structure learning and parameter learning. The structure of the $\mathrm{BN}$ is derived from the general model for the particular set of activities to be recognized, by:

1. Adding a link from each activity node, to the the limb nodes that are relevant.

2. Adding links between the limb nodes which require coordination.

3. Adding a link from each limb node to the corresponding motion nodes (ddd).

4. Adding a link from each motion node to the next (in time).

Given the structure, the parameters can be estimated from training data. We consider a supervised learning scheme in which the parameters are obtained from a set of video sequences for each activity. There are 3 set of parameters: (i) prior probabilities for each activity, $P\left(A_{i}\right)$; (ii) probability of a limb node given the related activities and limbs, $P\left(L_{i} \mid A_{1: n_{i}}, L_{1: m_{i}}\right)$; and (iii) probability of each motion node given the corresponding limb and previous motion node, $P\left(D_{i} \mid L_{j}, D_{i-1}\right)$. Where: $A_{1: n_{i}}$, is set of $n_{i}$ activities that influence a limb variable; $L_{1: m_{i}}$, is the set of $m_{i}$ limb nodes that influence a limb node. Parameter learning was done using a MAP estimator with uniform Dirichlet priors.

\subsection{Recognition}

Recognition consists in obtaining the most probable activities given the motion data. We consider two alternative schemes. In the first scheme we obtain the posterior probability of each activity given the motion information: $P\left(A_{i} \mid D_{1: k, 1: m}\right)$, where $D_{1: k, 1: m}$ represents the set of $m$ motion variables for each of the $k$ limbs. These probabilities are obtained via probability propagation in the BN. The activities whose posterior probability is above certain threshold, $T$, are the recognized set, $R$ :

$$
R=\left\{A_{i}\right\}, \leftrightarrow P\left(A_{i} \mid D_{1: k, 1: m}\right)>T
$$

By changing this threshold, we can make a trade off between recall (fraction of labeled activities) vs. precision (fraction of windows that are labeled correctly). For the second scheme, we obtain the most probable explanation (MPE) set given the evidence. This corresponds to total abduction in BNs, the configuration(s) of maximum probability containing all the unobserved variables. That is:

$$
M P E=\operatorname{argmax}_{\mathbf{A}, \mathbf{L}} P\left(\mathbf{A}, \mathbf{L} \mid D_{1: k, 1: m}\right)
$$

where $\mathbf{A}, \mathbf{L}$ denotes the set of activity and limb nodes in the network. So in this way we obtain the set of activities which have a higher probability given the data. Thus, the recognized set, $R$, consists of all the activities that have value "yes" in the MPE set:

$$
R=\left\{A_{i}\right\}, \leftrightarrow A_{i}=\text { yes } \in M P E
$$

The inference mechanism is applied to each window in the sequence, obtaining $R$. For the experiments, we used a stochastic simulation algorithm [5] for probability propagation, and Nilsson's algorithm [7] for total abduction. 


\section{Experimental Results}

To test the proposed methodology for activity recognition, we considered the 6 types of activities previously described, using the recognition network shown in figure 3. We used videos with a black background and with people wearing color landmarks. The camera is fixed and captures the persons facing the camera or in profile. We trained the network with 175 samples of 7 persons performing 6 types of activities. The test activities were selected to include different conditions. The sampling rate was 15 images per second, with 15 frames per window and an overlap of 12 frames. We tested both inference schemes for recognition, with a set of 50 different sequences. In the test set, 5 of the sequences consisted of two activities that were performed simultaneously. Recognition was performed continuously, so each sequence is part of a video in which a person can change activity at any time.

We used the precision-recall rates for quantitative evaluation. There is a trade off between recall and precision that depends on the recognition threshold. If we set the threshold to 0.6 , the results are summarized in table 1 for both inference schemes. We notice a better recall using abduction, and similar precisions with both schemes. For the cases that were labeled incorrectly, only 2 (for both schemes) were labeled with a different activity, the other were partial matches (of two simultaneous activities, one was recognized).

We used the precision-recall framework to assess the performance with respect to the recognition threshold for probability propagation. We obtain a high recognition rate, above $93 \%$, for a wide range of different tresholds (the recall varies from 0.4 to 1 ). In general, the model correctly recognizes the activities or it is indecisive; in very few cases it confuses the activities. The misclassified sequences occur mainly when there is a transition between different activities in the continuous sequence. Some of the unlabeled windows are between correctly labeled ones, so by taking into account the labels from neighbor windows, the problem could be reduced.

\section{Conclusions and Future Work}

We have developed a general model for human activity recognition. The motion of each limb and the coordination between them is considered in a layered Bayesian network that can represent and recognize a wide range of human activities. It can recognize continuous and simultaneous activities, as well as handle occlusions and missing data. We explore two inference methods: probability

Table 1. Precision-recall for probability propagation (PP) and total abduction (TA).

\begin{tabular}{|c|c|c|c|}
\hline & No. of seqs. & Recall & Precision \\
\hline \hline Test set (PP) & 50 & $84 \%$ & $93 \%$ \\
\hline Test set (TA) & 50 & $94 \%$ & $91.5 \%$ \\
\hline
\end{tabular}


propagation and abduction. The model was tested with video sequences of different people performing 6 activities. The recall-precision rate was evaluated with good results for both inference schemes. In the future, we plan to avoid the use of markers on the limbs. We will also take into account the information from previous windows, by building a dynamical model on top of the classifier.

\section{References}

1. Ali, A. and Aggarwal J. K., Segmentation and Recognition of Continuous Human Activity, Proceedings of the IEEE Workshop on Detection and Recognition of Events in Video (2001)

2. Aviles-Arriaga, H., Sucar, L.E. Dynamical Bayesian Networks for Visual Recognition of Dynamic Gestures, Journal of Intelligent and Fuzzy Systems, Vol. 12 (2002) 243-250.

3. Bohm, K., Broll, W., Sokolewicz, M. Dynamic Gesture Recognition Using Neural Networks; A Fundament for Advanced Interaction Construction, SPIE Conference on Electronic Imaging, Science and Technology, San Jose California, USA (1994)

4. Jones, M.J. and Rehg, J.M. Statistical Color Models with Application to Skin Detection, Computer Vision and Pattern Recognition (1999) 274-280

5. Kanazawa, J., Koller, D. and Russell, S. Stochastic Simulation Algorithms for Dynamic Probabilistic Networks, Proceedings of the 11th Annual Conference on Uncertainty in Artificial Intelligence (1995)

6. Kjaerulff, U. A Computational Scheme for Dynamic Bayesian Networks, Department of Mathematics and Computer Science, Institute for Electronic Systems, Aalborg University. Denmark (1993)

7. Nilsson, D. An Efficient Algorithm for Finding the M Most Probable Configurations in Bayesian Networks. Statistics and Computing, Vol. 9 (1998) 159-173.

8. Polana, R., Nelson, R. Detection and Recognition of Periodic, Nonrigid Motion. International Journal of Computer Vision, Vol. 23 (1997) 261-282.

9. Rao, C., Shah, M. View-Invariance in Action Recognition. IEEE Computer Vision and Pattern Recognition (2001) 316-321.

10. Vogler, C. and Metaxas, D. Parallel Hidden Markov Models for American Sign Language Recognition. Proceedings of the International Conference on Computer Vision (1999)

11. Yaacoob, Y., Black, M.J. Parameterized Modeling and Recognition of Activities. Computer Vision and Image Understanding, Vol. 73 (1999) 232-247.

12. Zelnik-Manor, L. Irani, M. Event-Based Analysis of Video. IEEE Computer Vision and Pattern Recognition (2001) 